\title{
Development and Application for Intelligent Monitoring System of Concrete Temperature Control
}

\author{
Lei Zhang ${ }^{1}$, Guoxin Zhang, ${ }^{1, *}$ Yi Liu' ${ }^{1}$ Xiangming Deng ${ }^{2}$ and Liying Yang ${ }^{2}$ \\ ${ }^{1}$ State Key Laboratory of Simulation and Regulation of Water Cycle in River Basin, China Institute of Water Resources and \\ Hydropower Research, Beijing, China \\ ${ }^{2}$ Wudu Water Diversion Project Construction and Management Bureau of Sichuan Mianyang, Sichuan, China \\ ${ }^{*}$ Corresponding author
}

\begin{abstract}
The temperature control and crack prevention of the high dam is with very much difficulties. One important reason is that the unsmooth information causes improper measures and management, that is "four don't" for obtaining the informationnot timely, not accurate, not true, not systematic; furthermore, "four top" problems during the temperature control construction process can't be controlled easily, that is: large temperature difference, large scale of drop in temperature, high rate of temperature fall, large temperature gradient, which could finally cause the generation of distress in concrete. In order to solve "four don't" to control "four tops", take informationized, automatic and intelligent technical methods, establish individual ideal temperature control curve model, temperature and flow prediction and forecasting model, temperature control effectiveness evaluation model, crack risk alarm model and other online real-time analysis models, develops key technology and processes for simulative analysis of the whole process of the whole dam, develops pervasive, stable and highly precise automatic monitoring and controlling instrument and equipment, researches and develops all software function models and subsystems, integrates a complete digital concrete temperature control intelligent monitoring system for Huangdeng Dam, realizes comprehensive, accurate and timely automatic collection of large quantity of temperature control information and relevant key factors, realizes automatic analysis, evaluation and warning and alarm as well as support of intervening measure decision for large quantity of temperature control information, realizes intelligent water cooling of the whole dam under the "nonmanual intervention" ideal conditions, and improves the water cooling construction quality while reducing the construction error rate. The system dynamically and high-efficiently manages, real-timely controls and analyzes the temperature control information of Huangdeng Hydropower Dam with many characters, such as real-time, continuity, automation and intelligentization, etc., it effectively manages all links during the temperature control process of the dam, realizes online real-time monitoring and feedback control of construction, which is able to effectively solve "four don't" and control "four tops", basically realizes the temperature control and crack prevention of concrete, and improves construction quality and informatization control level of Huangdeng Hydropower Station.
\end{abstract}

Keywords-intelligent monitoring; temperature control and crack prevention; digital Huangdeng; informatization; concrete dam

\section{INTRODUCTION}

The dam body of the roller-compacted concrete gravity dam of Huangdeng Hydropower Station is 203m high, its maximum bottom width is more than $160 \mathrm{~m}$, and it is the highest roller-compacted concrete gravity dam at present in China; the Huangdeng dam is with large volume of pouring of concrete, high construction strength, long construction period, large concrete construction surface, the water temperature and climate conditions are awful, and the temperature control and crack prevention[1-3] of the dam body is with very much difficulties. The reasons for distress in concrete are very complex, which are mainly on the aspects of structures, materials and construction. One important reason is that the unsmooth information causes improper measures and management, that is "four don't" for obtaining the informationnot timely, not accurate, not true, not systematic. Furthermore, basic data parameters in design stage differ considerably from actual parameters sometimes, the design need to be optimized and adjusted constantly; in the construction stage, the construction quality is often affected by the quality of the field engineering staff, resulting in larger deviation from the design state, which leads to the "four tops" problems of temperature control construction, that is: large temperature difference, large scale of drop in temperature, high rate of temperature fall, large temperature gradient, which could finally cause the generation of distress in concrete.

The implementation of intelligent monitoring system[4,5,7] of concrete temperature control of digital Huangdeng dam dynamically and high-efficiently manages, real-timely controls and analyzes the temperature control information of Huangdeng Hydropower Dam with many characters, such as real-time, continuity, automation and intelligentization, etc., it effectively manages all links during the temperature control process of the dam, realizes online real-time monitoring and feedback control of construction, which is able to effectively solve "four don't" and control "four tops", basically realizes the temperature control and crack prevention of concrete, and improves construction quality and informatization control level of Huangdeng Hydropower Station.

\section{SySTEM ARCHITECTURE AND MAIN FUNCTIONS}

Similar to artificial intelligence, an intelligent Monitoring system is composed of such four parts as perception, interconnection, analysis \& decision-making, and control. 
"Perception" means (automatic and manual) acquisition of various key factors; "Interconnection" aims to achieve multilevel network communications, and close link, interflow and sharing between various remote and heterogeneous terminals, and software and hardware resources by means of informatization. Control consists of artificial intervention and intelligent control. The former refers to human intervention based on commands of early warning, alarm and feedback of diversified solutions and measures that are formed on the basis of intelligent analysis, judgement and decision-making. The latter refers to automatic and intelligent control of microenvironment indicators covering temperature, humidity and wind speed, concrete preservation and pipe cooling regulation. "Analysis \& decision-making", as the core of the system, enable the formation of a decision after a process covering learning, memorization, analysis, judgement, inversion and prediction. "Perception", "Interconnection" and "Control" depend on and supplement each other so as to form a unified whole of intelligent monitoring encompassing "analysis \& decision-making”, as shown in Figure I.

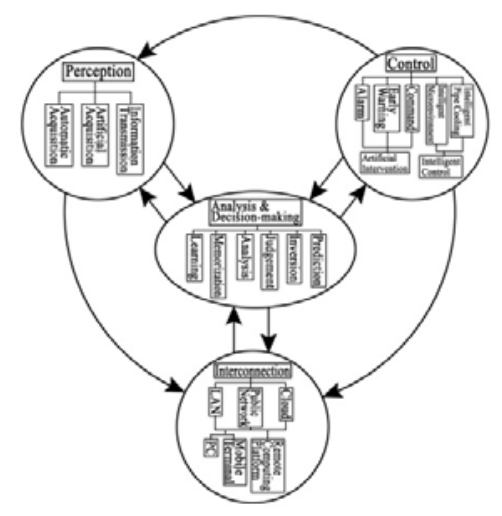

FIGURE I. STRUCTURE OF INTELLIGENT MONITORING ON MASS CONCRETE

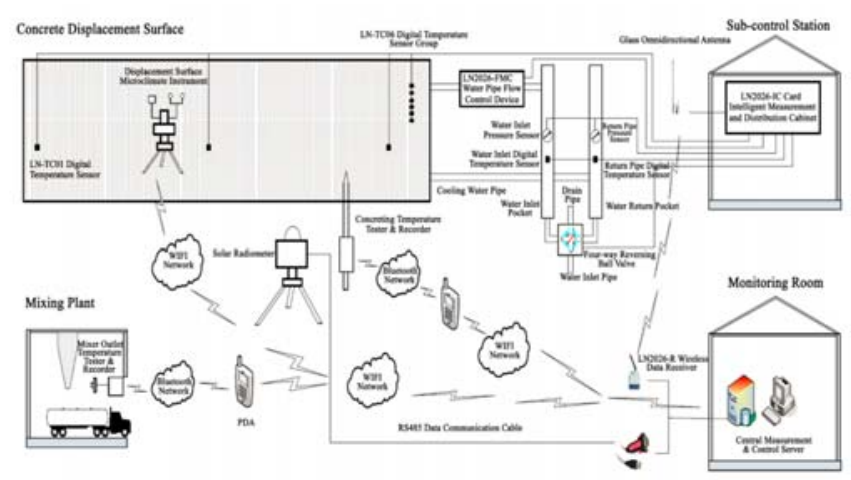

FIGURE II. SCHEMATIC DIAGRAM OF SITE STRUCTURE OF INTELLIGENT MONITORING SYSTEM

The "Intelligent monitoring" system is composed of such two levels as "surveillance" and "control”. The former refers to comprehensive test, detection and capture of information in various construction processes affecting temperature and crack control and prevention by taking advantage of the perception and interconnection functions. The latter refers to intelligent control or artificial intervention with factors affecting temperature. FigureII shows the schematic diagram of the site structure of the intelligent monitoring system for concrete crack prevention. In various concrete construction links, sensors are distributed at the mixing plant, concreting displacement surface, interior and surface of concrete. Sub-control stations are arranged in the dam area as required for collecting relevant information and giving commands. Intelligent control is applied to any parameter that allows automatic control. All subcontrol stations achieve information exchange with the master control room by means of wireless transmission, thus forming a complete monitoring system.

Intelligent monitoring system of concrete temperature control for digital Huangdeng dam : takes mass concrete crack prevention as the fundamental purpose, uses automatic monitoring technology, GPS technology, wireless transmission technology, network and database technology, data mining technology, numerical simulation technology, automatic control technology to achieve information real-time acquisition, real-time transmission, automatic management, automatic evaluation, automatic analysis, real-time monitoring and realtime feedback control of temperature control and other dynamic intelligent monitoring, analysis and control system of temperature control construction. It mainly includes two software subsystems under digital Huangdeng platform: information monitoring and acquisition of temperature control, intelligent temperature control, Figure III shows the system architecture diagram, and the system implements the following functions:

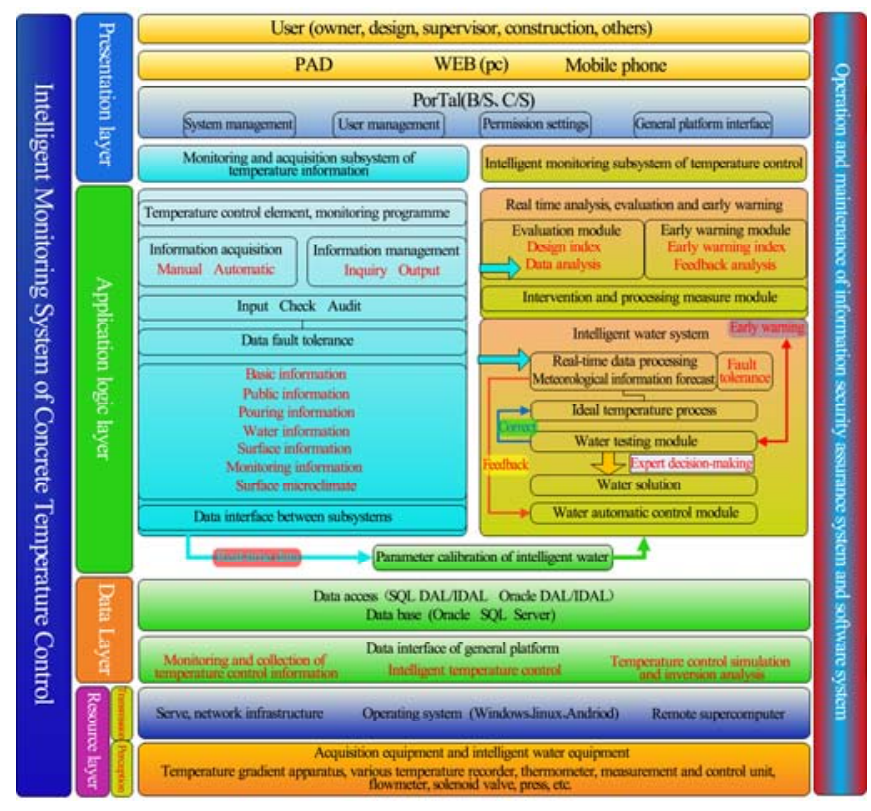

FIGURE III. SYSTEM ARCHITECTURE DIAGRAM OF INTELLIGENT MONITORING SYSTEM OF CONCRETE TEMPERATURE CONTROL FOR DIGITAL HUANGDENG DAM

(1) Realize automatic acquisition of temperature control elements such as, aggregate temperature, outlet temperature, placing temperature, pouring temperature, surface microclimate, internal temperature process of the concrete, temperature gradient, cooling water inlet water temperature, outlet water 
temperature, water flow and so on, realize automatic acquisition of full temperature control information the maximum possible to ensure that the data in real time and accurate;(2)Put forward 12 evaluation indexes and the method for determining the threshold value of temperature control construction quality based on statistical analysis and crack risk assessment method based on simulative analysis of the whole process of the whole dam, to realize the automatic evaluation and early warning based on the real-time monitoring information, and the decision support of the intervention measures, the evaluation, early warning and intervention more timely compared with delayed evaluation and periodic forecast adopted by Xiluodu and other engineering at present;(3) Realizes intelligent water cooling of the whole dam under the "non-manual intervention" ideal conditions, and improves the water cooling construction quality while reducing the construction error rate;(4)Realize sharing of temperature control construction, monitoring, evaluation, and data of early warning, to provide the basis for site construction management.

\section{R\&D OF KEY TECHNOLOGY AND SYSTEM}

The main functions of intelligent monitoring system of concrete temperature control of digital Huangdeng dam include real-time acquisition and transmission of temperature control information, efficient management and visualization of temperature control information, simulative analysis and back analysis of temperature stress, effect evaluation and early warning of temperature control construction, intelligent control of temperature control construction and so on, each function module has the corresponding key methods and technical support, On this basis, the corresponding software system and hardware equipment are developed, and integrates a complete intelligent monitoring system.

A. Key Technology and Processes for Whole Process Simulative Analysis of Whole Dam

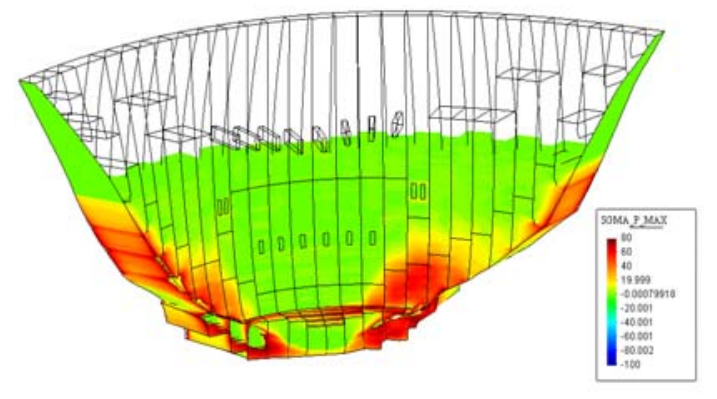

FIGURE IV. RESULTS OF TEMPERATURE AND STRESS SIMULATION FEEDBACK ANALYSIS

The technology and processes can simulate nine processes such as the concrete pouring, excavation, hardening, temperature control, arch closure, water storage, environmental change, long-term operation and so on, can do coupling analysis of temperature, seepage and stress and so on, with elastic-plastic and damage nonlinear analysis model and considering open close iteration of seam to realize the accurate simulation of various seam states, realize real-time tracking simulative analysis[6,8] for whole process from pouring of dam to operation, promptly forecast the temperature, stress and crack risk, put forward measures and suggestions. Furthermore, based on the simulation model of the whole process of the whole dam and multiple and large quantity of monitoring elements, carry out back analysis on the simulation parameters of the dam's working performance, study real working state of dam during construction period, initial storage period and operation period, set up the corresponding early warning index and threshold value, realize real working state analysis and back analysis on concrete dam. FigureIV shows results of temperature and stress simulation feedback analysis.

\section{B. Determining Method for Individualized Ideal Temperature Control Curve}

Under the condition of certain temperature control standard, considering the characteristics of different dam types and different sections of the dam, according to the principle of minimum temperature stress, the individual temperature control curve is established from the factors of temperature difference classification, cooling rate, spatial gradient control and so on, FigureV is Standard Cooling Process Curve. It is necessary to carry out sensitivity analysis on various control parameters that may affect the course of temperature change before the construction of the dam, fully know about the potential influence of variation of different parameters of the dam on the temperature drop process and temperature stress, according to the results of the sensitivity analysis, to develop the control standards that should be followed in the pouring of concrete in different sections of the dam in different seasons, such as control standards for basic temperature difference, temperature difference between upper and lower layers, temperature difference between inside and outside and so on. On this basis, obtain ideal temperature drop curve of risk minimization which is suitable for this type of dam, at the same time, the results of all kinds of sensitivity analysis can provide important theoretical basis for optimization of various possible emergency control measures during the actual cooling control process.

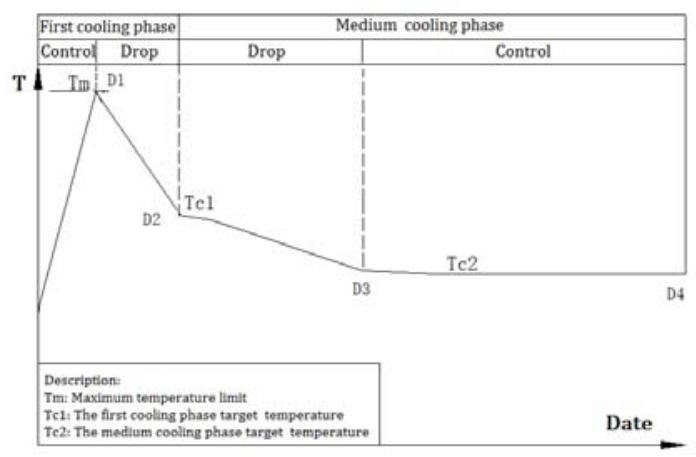

FIGURE V. STANDARD COOLING PROCESS CURVE

\section{Prediction and Forecasting Model of Temperature and Flow}

This model can predict the future changes of temperature, give water control parameters. It includes internal heat, surface heat dispersion, heat transfer between adjacent blocks, water with heat and other factors, use monitoring data to make self- 
learning and self-correction, the average precision of the model can be within $0.1^{\circ} \mathrm{C}$; and can conduct prediction and forecast for the solution of water parameters (flow, water temperature, flow direction) according to the process of actual temperature measurement, individual temperature control curve and prediction results of temperature, based on simulation calculation model, combine with the measured data

The prediction and forecast of water solution is based on theoretical calculation, at the same time, can consider a variety of concrete heating models according to the site conditions, further adopt neural network and other uncertainty methods, to carry out continuous iterative correction for prediction model, it will be more accurate with more measured data and longer time of water, which is with intelligent learning function.

\section{Evaluation Model of Temperature Control Effectiveness}

For the large number of monitoring data automatically acquired in construction process of mass concrete, such as air temperature, aggregate temperature, outlet temperature, placing temperature, pouring temperature, cooling water flow and water temperature, internal temperature of concrete and other temperature control elements, the module can make direct, realtime, comprehensive and quantitative evaluation for these temperature control elements with limited 8 tables and 12 figures, and quickly determine whether the temperature control measures at present need to be modified the adjusted. FigureVI is Comprehensive Curve Chart, which can track and evaluate the internal temperature variation, water situation and temperature change situation.

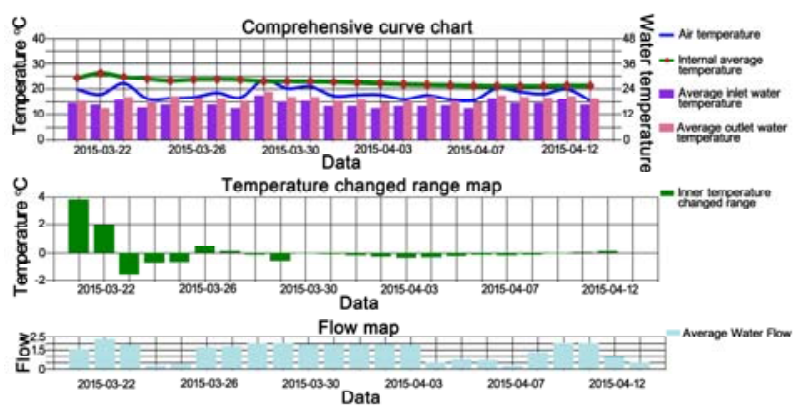

FIGURE VI. TRACKING AND EVALUATION OF TEMPERATURE CONTROL EFFECT

\section{E. Hardware R\&D of Intelligent Temperature Control and Measurement Control}

To achieve the goal of intelligent temperature control in the whole process, it is necessary to develop corresponding measurement and control hardware equipment, as shown in FigureVII, which mainly includes: have developed monitoring equipments of digital temperature, water pressure, temperature gradient, surface microclimate that adapt to the harsh environment of dam construction, successfully realize automatic acquisition of large quantity of temperature control data; have developed portable temperature acquisition equipment that adapt to the mobile needs in construction site, based on GPS and Bluetooth technology, have realized realtime temperature measurement, measuring point location, transmission and storage; have developed large capacity data real-time transmission device which integrating RS485, ZigBee, WIFI and other means, which can meet the demand of large quantity of data network transmission under the bad condition; have developed high precision digital measurement and control unit, flow measurement and control device and a reversing device, which and realize automatic measurement and control of all kinds of temperature (internal temperature, air temperature, water temperature, etc.), flow, flow direction.

\section{F. System Development and Integration}

Intelligent monitoring system of concrete temperature control of digital Huangdeng dam mainly includes monitoring and acquisition subsystem of temperature control information, management, evaluation and early warning subsystem of concrete temperature control information and intelligent automatic water subsystem of temperature control these three software subsystem. For the problems of temperature control construction monitoring on mass concrete, the research team led by the China Institute of Water Resources and Hydropower Research has carried out a comprehensive study on the theoretical method, analysis model, hardware equipment, software system and other aspects for intelligent temperature control on mass concrete, the whole system have spent more than 2 years from the program design, software development, hardware development, interface development, hardware and software integration, field experiments to formal application, eventually formed a complete temperature control technology of mass concrete crack control and intelligent integrated system.

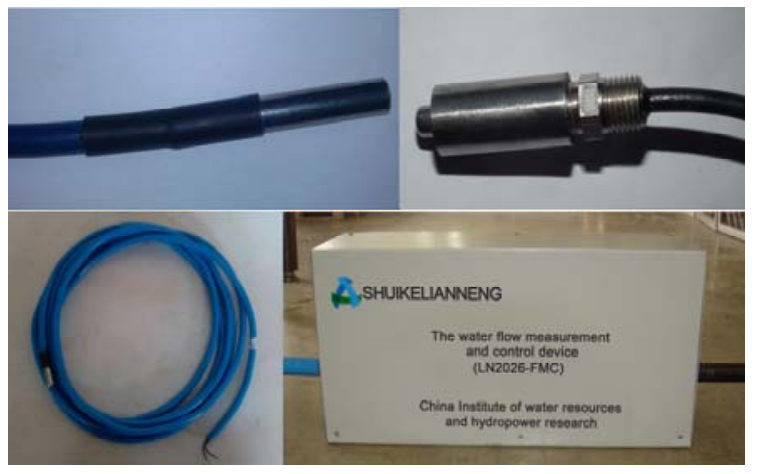

FIGURE VII. R\&D OF HARDWARE EQUIPMENT

\section{APPLICATION AND EFFECT}

The system has been put into operation since March 2015, in Huangdeng Hydropower Station, it have completed the realtime monitoring of 2.1 million cubic meters of concrete up to now, no temperature crack is found at present. The system can carry out real-time monitoring for Pouring of concrete of dam, such as pouring strength, pouring amount, pouring progress and so on, carry out automatic monitoring for meteorological information in dam area and surface, such as temperature, humidity and wind speed and so on, carry out real-time monitoring and evaluation and early warning feedback for temperature control factors in concrete production process, such as the temperature of aggregate, outlet, placing and pouring, carry out real-time monitoring for internal temperature of concrete and temperature gradient and so on, carry out real- 
time monitoring for water cooling conditions including temperature, flow, flow direction, pressure and so on, make analysis and evaluation on temperature rise of concrete in dam, make real-time tracking and feedback of the temperature and stress of the whole dam, the system has successfully realized comprehensive and accurate automatic acquisition of temperature control information, real-time evaluation, intelligent warning, intelligent control of water and decision support, improving the quality of temperature control, saving manpower; which effectively improve the management level of concrete construction. FigureVIII is Figure of Water Cooling Effect.

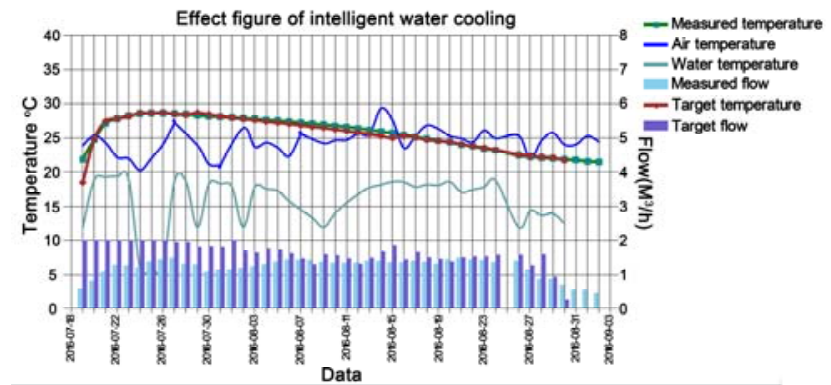

FIGURE VIII. EFFECT FIGURE OF INTELLIGENT WATER COOLING

\section{SUMMARY}

The application of intelligent temperature control system of mass concrete in the Huangdeng Hydropower Station realizes dynamical and high-efficient integrated management real-time control and analysis on temperature control information of dam, and with many characters, such as real-time, continuity, automation and intelligentization, etc., it effectively manages all links during the temperature control process of the dam, realizes online real-time monitoring and feedback control of construction, and realizes the various engineering information integration and data sharing of dam; in the whole life cycle of the project construction, realizes the dynamic updating and maintenance of the integrated information, provides information application and support platform for quality and process control in construction process of Huangdeng Hydropower Station, improves the management efficiency and information management level of the construction project of Huangdeng Hydropower Station.

Some results of research on the key technology of dynamic intelligent temperature control of mass concrete crack prevention have been successfully applied to a number of large-scale water conservancy and hydropower projects, and have obtained more than 20 patents and a number of software copyrights, it is innovative in theory and method, has made a breakthrough in key technology, and has remarkable economic and social benefits, has good prospect for application.

\section{ACKNOWLEDGMENT}

The research was supported by the National Key Research and Development Project of China (Grant NO. 2016YFB0201000), the National Key Basic Research Program of China (Grant NO. 2013CB036406, 2013CB035904), the National Natural Science Foundation of China (Grant NO.
51579252, 51439005), the Special Scientific Research Project of the State Key Laboratory of Simulation and Regulation of Water Cycle in River Basin and the Special Scientific Research Project of the China Institute of Water Resources and Hydropower Research（Grant NO. SS0145B392016）.

\section{REFERENCES}

[1] Zhang Guoxin, Liu Yi, Li Songhui,et al. Research and practice of nine three one temperature control mode[J]. Journal of Hydroelectric Engineering: 2014, 33(2): 179-184.(in Chinese)

[2] Thermal stress and temperature control of mass concrete[M]. Beijing: China electric power press, 1999.

[3] Liu Yi, Zhang Guoxin. Discussion on key points of temperature control and crack prevention dam[J].Water Resources and Hydropower Engineering: 2014, 45(1): 77-89. (in Chinese)

[4] Zhu Bofang, Zhang Guoxin, Jia Jinsheng,et al. Numerical monitoring of concrete dams - a new way for improving the safety control of concrete dams[J]. Journal of Hydroelectric Engineering: 2009,28(1):130-136.(in Chinese)

[5] Liu Yi,Zhang Guoxin,Wang Jimin,et al.Numerical monitoring methods and system for construction of super high arch dam and its engineering practice[J]. Water Resources and Hydropower Engineering: 2012, 43 (3): 33-37.(in Chinese)

[6] Zhang Guoxin, Zhang lei, Liu Yi, etc. The True Working Performance Inversion Simulation Analysis of Jinping Level-I Arch Dam[C]. Chen Yunhua//Key technical issues and research progress in hydropower development. Zhengzhou: The yellow river water conservancy press. 2014.77-83

[7] Zhang Guoxin, Liu Youzhi, Liu Yi. "Digital dam" to "intelligent dam"Progress of temperature control research on high dam[C]//Jia Jinsheng, Chen Yunhua. Technical progress in construction and management of dams. Zhengzhou: Zhengzhou: The yellow river water conservancy press. 2012.74-84

[8] Lei Zhang, Yi Liu,Bingqi Li etc.. Study On Real-time Simulation Analysis and Inverse Analysis System for Temperature and Stress of Concrete Dam, Mathematical Problems in Engineering, Volume 2015, Article ID 306165 